\title{
États généraux de la bioéthique
}

Une perspective sociologique

Séverine Mathieu

«Quel monde voulons-nous pour demain? : c'est autour de cette question que, durant le premier semestre 2018, ont été organisés sous l'égide du Comité consultatif national d'éthique pour les sciences de la vie et de la santé $(C C N \varepsilon)^{l}$, les États généraux de la bioéthique ${ }^{2}$ inaugurant les débats sur la prochaine révision des lois de bioéthique ${ }^{3}$. Leur vocation était d'animer le débat public et d'éclairer le gouvernement qui devrait prochainement soumettre au législateur un projet de loi.

Lorsque le 25 septembre 2018 , le CCNE rend public son avis $129^{4}$ et se prononce en faveur de l'ouverture de la PMA (procréation médicalement assistée, parfois nommée AMP, assistance médicale à la procréation) aux femmes seules et aux couples de femmes (comme il l'avait déjà fait dans son avis 126 du 15 juin 2017 intitulé «Avis du CCNE sur les demandes sociétales de recours à l'assistance médicale à la procréation »), les oppositions s'expriment rapidement. Le soir même,

Vignette (Photo @ Inserm - Patrick Delapierre).

${ }^{1}$ Le CCNE pour les sciences de la vie et de la santé a été créé le 23 février 1983 par décret présidentiel ( $\left.n^{\circ} 83-132\right)$. II a pour mission de «donner son avis sur les problèmes moraux qui sont soulevés par la recherche dans les domaines de la biologie, de la médecine et de la santé, que ces problèmes concernent l'homme, des groupes sociaux ou la société toute entière ». Cette mission a été reprécisée dans la Loi n 2004-800 du 6 août 2004 qui indique « Le Comité consultatif national d'éthique pour les sciences de la vie et de la santé a pour mission de donner des avis sur les problèmes éthiques et les questions de société soulevés par les progrès de la connaissance dans les domaines de la biologie, de la médecine et de la santé (Art. L. 1412-1.).

${ }^{2}$ https://etatsgenerauxdelabioethique.fr/

${ }^{3}$ Cette révision a été rendue obligatoire dès la loi de 2004 qui instituait le réexamen par le Parlement de la loi dans un délai de cinq ans (délai qui n’a pas été respecté). La loi de 2011 a élargi ce délai à 7 ans maximum. Cette loi stipule aussi que toute révision de la loi doit être précédée d'États généraux que le CCNE a la responsabilité d'organiser. Les derniers ont eu lieu entre le 18 janvier 2018 et le 30 avril 2018. Le CCNE a piloté le débat en s'appuyant sur les Espaces de réflexion éthique régionaux, un site web dédié et des auditions. Ces auditions ont concerné 154 associations d'usagers, groupes d'intérêt et sociétés savantes, ainsi que les représentants des cultes et des courants de pensée, ou encore les grandes institutions. Certaines auditions se sont poursuivies jusqu'au 24 mai 2018. Rappelons que la loi HurietSérusclat $n^{\circ} 88-1138$ du 20 décembre 1988 est considérée comme la première loi de bioéthique.

${ }^{4}$ Avis intitulé « Contribution du Comité consultatif national d'éthique à la révision de la loi de bioéthique 2018-2019» http://www.ccne-ethique.fr/sites/default/ files/avis_129_vf.pdf

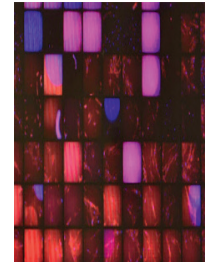

Sociologue, directrice d'études, École Pratique des Hautes Études (EPHE), Paris Sciences et Lettres (PSL), 4-14, rue Ferrus, 75014 Paris, France. severine.mathieu@ephe.psl.eu

la «Manif pour tous » manifeste devant les locaux du CCNE et le dimanche suivant, le 30 septembre, dans un entretien au Parisien, l'archevêque de Paris, Monseigneur Aupetit, invite les catholiques à se mobiliser par une parole contre cette extension, au nom du respect de la dignité humaine ${ }^{5}$. La déclaration de la Conférence des évêques de France publiée le 20 septembre ${ }^{6}$, avant donc la publication de l'avis 129, constitue quant à elle un texte documenté, argumenté et fidèle à ses principes. Mais que penser de toute cette mobilisation, qui peut surprendre dans le contexte français laïque?

On le sait, les évolutions très rapides de la science posent des questions qui mettent en conflit les représentations du vivant: quelle attitude avoir devant certaines innovations? Qu'autoriser? Qu'interdire? Que contrôler? À propos de thèmes aussi divers que le début et la fin de vie, la procréation médicalement assistée, l'intervention médicale sur le vivant, les discussions et questions sont multiples. De nombreux acteurs sociaux s'y intéressent : médecins, chercheurs, religieux, associations.

Les États généraux de 2018 ont voulu ouvrir le débat citoyen, dans un processus alliant échanges et souci du consensus. Mais ces débats ne participent-ils pas également à la formation des principes moraux en matière de bioéthique? Sur quelles bases établir un consensus?

\footnotetext{
5 https://www.leparisien.fr/societe/pma-gpa-fin-de-vie-mgr-aupetit-appelleles-catholiques-a-se-mobiliser-29-09-2018-7906764.php

${ }^{6}$ Déclaration intitulée « Respectons la dignité de la procréation! 》, https://eglise. catholique.fr/wp-content/uploads/sites/2/2018/09/180920-Synthese-declaration-la-dignite-de-la-procreation.pdf
} 
Quelle place pour les différentes religions ${ }^{7}$, les courants de pensée, les associations (familiales, $\mathrm{LGBT}^{8}$, féministes, citoyennes), les sociétés savantes dans l'élaboration de ces principes éthiques?

Depuis les années 1980, en France, les questions de bioéthique se sont constituées comme un enjeu social et politique d'importance [1] et se traduisent en 1994 dans la loi de bioéthique [2, 3] $(\rightarrow)$ puis par la création de l'Agence de la biomédecine en $2004^{9}$ En prévision de la seconde révision (initialement prévue pour 2010, et qui s'est traduite $(\rightarrow)$ Voir l'éditorial de H. Chneiweiss, $\mathrm{m} / \mathrm{s}$ $n^{\circ} 2$, février 2018, page 107 par la loi 2011-214 du 7 juillet 2011 ; la première ayant conduit à la loi 2004-800 du 6 août 2004) des premiers États généraux de la bioéthique, pilotés par Jean Léonetti, ont été organisés en 2009. Pour ce qui concerne la PMA, l'un des enjeux principaux est alors la question de la levée de l'anonymat des dons de gamète, qui sera maintenu [4]. Actuellement, la $P_{M A}{ }^{10}$ n'est pas ouverte aux femmes célibataires et aux couples de femmes; la gestation pour autrui (GPA) demeure interdite. En 2012, les virulents débats sur le «mariage pour tous », qui aboutiront à la promulgation de la loi n²013-404 (dite «Loi Taubira») du 17 mai 2013 ouvrant le mariage aux couples de personnes de même sexe, modifient la donne. Lors de la révision de 2011, des médecins ont déclaré publiquement avoir orienté des femmes vers des cliniques étrangères pour un recours à la PMA, et des témoignages de femmes seules ou homosexuelles se sont multipliés. La société a-t-elle évolué ?

Les tout récents débats organisés lors des États généraux de la bioéthique de 2018 ont abordé neuf thèmes: cellules-souches et recherche sur l'embryon humain; examen génétique et médecine génomique; dons et transplantations d'organes; neurosciences; données de santé ; intelligence artificielle et robotisation; santé et environnement; procréation et société ; prise en charge de la fin de vie. Le thème «procréation et société », objet de plus de la moitié des débats, sera l'objet d'un prochain billet, car il donne à voir la façon dont s'érigent des principes éthiques en matière procréative et familiale $[5,6]$. Les acteurs sociaux sont nombreux à vouloir contribuer à l'élaboration de ces principes, parmi lesquels les représentants des religions ${ }^{11}$, notamment catholique, dont l'institution souhaite réaffirmer son autorité morale sur ces questions $[7,8]$.

\footnotetext{
${ }^{7}$ Le CCNE comprend 39 membres dont cinq personnalités désignées par le Président de la République appartenant aux «principales familles philosophiques et spirituelles ». Le président du CCNE est nommé quant à lui pour une période de deux ans, renouvelable. Les 39 autres membres du CCNE sont nommés pour quatre ans.

${ }^{8}$ Le sigle LGBT désigne les personnes lesbiennes, gays, bisexuelles, et transgenres.

9 L'Agence de la biomédecine (ABM) est une agence publique nationale de l'État. Ses missions s'exercent dans 4 domaines : le prélèvement et la greffe d'organes et de tissus, le prélèvement et la greffe de cellules souches hématopoiétiques, l'assistance médicale à la procréation, l'embryologie et la génétique humaines. Elle délivre les autorisations de recherche sur l'embryon et les cellules souches embryonnaires, et s'assure donc que tous les prérequis mentionnés dans la loi, scientifiques (et pas seulement les progrès thérapeutiques majeurs) et éthiques, sont bien remplis. https://www.agence-biomedecine.fr/

10 « La procréation médicalement assistée a pour objet de remédier à l'infertilité d'un couple ou d'éviter la transmission à l'enfant ou à un membre du couple d'une maladie d'une particulière gravité. Le caractère pathologique de l'infertilité doit être médicalement diagnostiqué. L'homme et la femme formant le couple doivent être vivants, en âge de procréer et consentir préalablement au transfert des embryons ou à l'insémination », Article L2141-2 du Code de la santé publique de 1994, modifié par la loi 2011-814 du 7 juillet 2011 relative à la bioéthique.

${ }^{11}$ En 2018, des représentants des religions juive, musulmane, catholique et protestante ainsi que bouddhiste ont été auditionnés. Des représentants de courants libres penseurs et francs-maçons ont également été entendus.
}

La PMA est une question politiquement centrale, puisque Emmanuel Macron, lors de sa campagne présidentielle, s'est engagé à l'ouvrir aux femmes seules et/ ou homosexuelles. Si la société semble avoir fortement évolué sur cette question [9], certains groupes religieux et groupes conservateurs s'y opposent. À cet égard, quelle place les récents débats, qui ont voulu associer les « citoyens au processus de révision », ont-ils laissé à ces différents groupes? Comment, dans un espace démocratique, s'articulent débat citoyen et débat politique? Comment ces conservateurs et les autres participants se sont-ils fait entendre? Quel poids ontils eu? Et comment leur répondent ceux qui, parfois dans leurs rangs, sont favorables à l'élargissement des modalités d'accès à la PMA ? Comment a été entendue - ou pas - la parole religieuse, celle des institutions et celle des croyants, sur les questions de bioéthique ${ }^{12} \grave{A}$ l'intérieur des différentes confessions religieuses, comment s'expriment les divergences ? ${ }^{13}$ L'examen durant les prochains mois des débats citoyens, des débats parlementaires et des médias tentera d'apporter des éléments de réponse à ces questions. Mon prochain billet s'intéressera, à partir de la participation à certains de ces débats, aux prises de parole religieuses lors de ces États généraux de la bioéthique. $\diamond$

General assembly for bioethics in France: a sociological perspective

\section{LIENS D'INTÉRÊT}

L'auteur déclare n'avoir aucun lien d'intérêt concernant les données publiées dans cet article.

\section{RÉFÉRENCES}

1. Mehl D. Naître? La controverse bioéthique. Paris : Bayard, 1999:333-56.

2. http://www.ladocumentationfrancaise.fr/dossiers/bioethique/ historique-lois-bioethique.shtml

3. Chneiweiss H. Les États généraux de la bioéthique de 2018. Med Sci (Paris) $2018 ; 34: 107-8$.

\footnotetext{
12 Question que pose par exemple le Grand maître de l'Orient de France Philippe Foussier qui, dans son éditorial du numéro 144 de la revue Questions de santé et de bioéthique, souligne: "Quand tant de courants invoquent Dieu pour éclairer ou prétendre éclairer ces problématiques, nous invoquons l'Homme ». II s'inquiète dans ce texte des prises de parole des religions dans les débats bioéthiques, en particulier sur la fin de vie, où selon lui, «la vision des cultes s'est imposée ». https:// www.la-croix.com/Sciences-et-ethique/Ethique/reflexions-francs-macons-revision-lois-bioethique-2018-08-01-1200959181.

13 Pour ne prendre que deux exemples : trente-cinq pour cent des catholiques pratiquants se déclarent favorables à l'ouverture de la PMA aux femmes en couple selon le sondage de l'IFOP de décembre 2018. Dans la contribution écrite de sa «Commission éthique et société », rédigée à l'occasion des États généraux, la Fédération protestante de France (FPF) se déclare « réticente à l'ouverture de la PMA » mais souligne d'emblée que «l'opinion des protestants est très partagée » sur la question de l'ouverture de l'AMP et ne propose pas de position unanime (https:// etatsgenerauxdelabioethique. $\mathrm{fr} / \mathrm{media} /$ default/0001/01/9a752d03e098a4f312b6 $5 \mathrm{dl} 646442 f 9 a 166 \mathrm{~d} 6927 . p d f)$. Et certains pasteurs, tels Jean-Marie de Bourquenay ou Ruth Wolff-Bonsirven, se prononcent en faveur de l'ouverture de la PMA (https:// www.reforme.net/les-dossiers-reforme/les-protestants-et-la-gpapma/).
} 


\section{RÉFÉRENCES}

4. Théry I. Des humains comme des autres. Bioéthique, anonymat et genre du don. Paris Éditions de l'EHESS, 2010.

5. Mathieu S. L'enfant des possibles. AMP, éthique religion et filiation. Paris : Éditions de l'Atelier, 2013.

6. Porqueres I, Gené $\varepsilon$. Défis contemporains de la parenté. Paris : Éditions de l'EHESS, 2009.

7. Hervieu-Léger D. Catholicisme, la fin d'un monde? Paris : Bayard, 2003.

8. Béraud C, Portier P. Métamorphoses catholiques. Paris : Fondation Maison des Sciences de I'Homme, 2015.
9. Les Français et les questions liées à la bioéthique. Sondage Ifop (Institut français d'opinion publique) pour La Croix et le Forum européen de bioéthique, décembre 2018, https://www.ifop.com/wp-content/ uploads/2018/03/3938-1-study_file.pdf
TIRÉS À PART

S. Mathieu

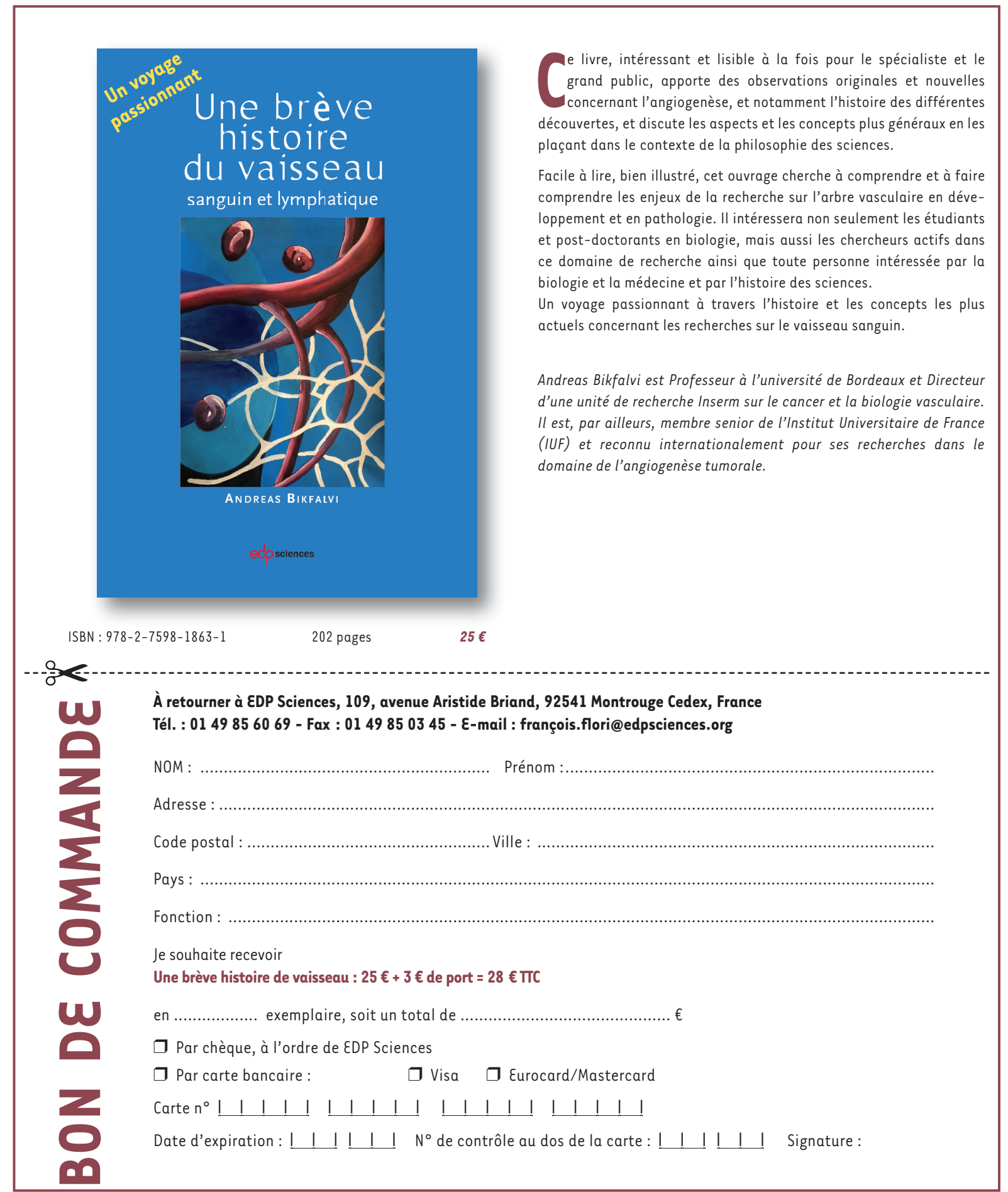

